\title{
Europäisch anschlussfähig werden
}

\author{
Peter Erath
}

Die Internationalisierung Sozialer Arbeit stellt hohe Anforderungen an das Management in sozialen Organisationen. Doch der Blick über den Zaun verspricht reiche Ernte: Mit europäischen Partnern kann Wissen kostengünstig generiert, transferiert und implementiert werden.

Dass die Soziale Arbeit auf allen Ebenen in einem noch nie da gewesenen Ausmaß europäischer und internationaler wird, lässt sich anhand vieler neuer Publikationen leicht belegen. Viel interessanter als diese Tatsache scheinen jedoch die unfreiwilligen Ursachen für diese neue Ausrichtung zu sein. Denn wenn aus der Sicht der kommunalen und regionalen Leistungsträger nur ein nationaler oder europäischer Markt mittels Konkurrenz und gestützt auf Rankings und Benchmarks dazu beitragen kann, die einzelnen Leistungsangebote besser, wirksamer und effizienter zu machen, dann bleibt wiederum der Anbieterseite gar nichts anderes übrig, als sich bei der Suche nach Best-practice-Modellen außer Landes zu begeben und sich diese entweder via Wissenstransfer einzukaufen oder per Kooperation einzuverleiben.

\section{Kommunikationsprobleme}

Vorbereitet wurde diese Entwicklung durch die zunehmende Distanzierung der (modernen) Gesellschaft gegenüber den sozial Tätigen. Jene zeichnet sich bekanntermaßen durch Komplexität aus und zeigt sich

dementsprechend vor allem an der Erfüllung der jeweiligen Funktion der einzelnen Teilsysteme (hier: Inklusionsvermittlung und Exklusionsvermeidung) und damit am Erfolg von Interventionen interessiert und nicht länger an den Motiven derer, die diese Funktionen als Träger organisieren oder als Mitarbeiterinnen und Mitarbeiter umsetzen.

Dabei belegen die Leitsprüche vieler (meist verbandlicher) Anbieter (»Wir sind für den Menschen da!« »Schwanger - wir sind an Ihrer Seite! «) nach wie vor das Vorhandensein eines eigenartigen intrasystemischen Leistungsverständnisses. Anstatt den Klienten und Leistungsempfängern das zuzusichern, was leistbar wäre (jedoch leider nicht immer geboten wird), nämlich eine freundliche und kompetente Betreuung und Unterstützung, wird versprochen, was gerade nicht geleistet werden kann. Denn nur Ehepartner, Freunde, Verwandte, Nachbarn können für den »Menschen « da sein, nicht die Professionellen! Viele Anbieter gehen offensichtlich immer noch davon aus, dass die Nutzer vorrangig am Motiv des Hel-

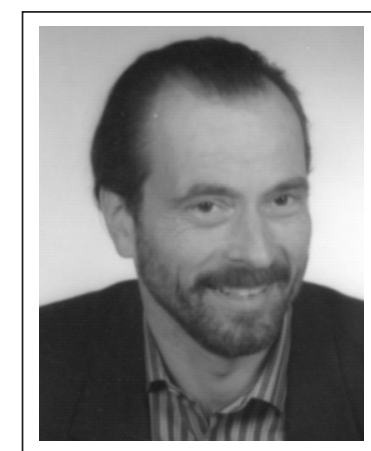

Der in Eichstätt lebende Sozialarbeitswissenschaftler Prof. Dr. Peter Erath ist an der Fakultät für Soziale Arbeit der Katholischen Universität Eichstätt-Ingolstadt im Diplomstudiengang »Soziale Arbeit « und als Beauftragter für den Masterstudiengang »European Social

Work « tätig. Schwerpunkte seiner wissenschaftlichen Arbeit sind die Theorien der Sozialen Arbeit sowie die Soziale Arbeit in Europa. Drittmittelforschung betreibt er vor allem im Bereich »Aufbau von Qualitätsmanagementsystemen «. Neuere Buchveröffentlichungen sind zur Weiterentwicklung der Sozialen Arbeit in Deutschland und Europa (in englischer Sprache) erschienen. Erath ist Mitglied der Deutschen Gesellschaft für Soziale Arbeit e. V. und des Fachausschusses Wirtschafts-, Rechts- und Sozialwissenschaften der Akkreditierungsgesellschaft ACUQIN e. V. sowie seit 2001 Privatdozent an der Universität Kuopio in Finnland.

E-Mail peter.erath@ku-eichstaett.de

fers interessiert sind und der eigentlichen (Hilfe-)Leistung offensichtlich eine nachrangige Bedeutung zumisst. Ein verhängnisvoller Irrtum!

Häufig nicht besser wird argumentiert, wenn es um Fragen der Wirksamkeit beziehungsweise des Erfolgs sozialer Hilfen geht. Im Rahmen einer intersystemischen Argumentation ist man sich dann schnell darin einig, dass es die anderen Teilsysteme der Gesellschaft - das politische System, das ökonomische System, das Erziehungssystem, das Strafvollzugssystem etc. - sind, die eine moralische Schuld am Vorhandensein und an der Unüberwindbarkeit sozialer Probleme tragen. Das unvermeidbare eigene Versagen wird dabei leicht übersehen.

Beide häufig zu beobachtenden (intra- und intersystemischen) Strategien tragen wenig zur Lösung der anstehenden Probleme, viel aber zur Immunisierung gegenüber zwei Erkenntnissen bei, die im Laufe der letzten zwanzig Jahre zunehmend rezipiert worden sind:

- Die Praxis der Sozialarbeit kann aus verschiedenen Gründen niemals zu 100 Prozent erfolgreich sein. Der 
Grund dafür liegt in der Tatsache, dass aufgrund verschiedener theoretischer Überlegungen der Erfolg einer Hilfeleistung nicht methodisch »erzwungen «, sondern immer nur »Zufall « sein kann. Sozialarbeit ist demnach durch eine Dilemmastruktur gekennzeichnet, die insbesondere durch quantitative Unbestimmbarkeit, doppeltes Mandat, fehlende Integrationskraft, Effizienzverdacht und Technologiedefizit gekennzeichnet ist (vgl. Erath 2006).
- Die moderne Gesellschaft ist nur noch äußert begrenzt in der Lage, beispielsweise mithilfe des Funktionssystems Politik die übrigen Funktionssysteme so zu bändigen, dass die Ausgewogenheit einer pluralistisch angelegten und sozial ausgerichteten Gesellschaftsordnung nicht aus den Fugen geraten kann. So hat die gesellschaftliche Modernisierung nicht nur zur Verbesserung von Lebensverhältnissen, sondern auch zu Individualisierung und damit verbundenen steigenden Exklusions-

\section{BEISPIELE GUTER PRAXIS: POLEN}

\section{Die Barka-Stiftung baut ein alternatives Unter- stuitzungssystem auf}

Die letzten 20 Jahre des vergangenen Jahrhunderts bewirkten in Polen Veränderungen in allen wichtigen politischen, wirtschaftlichen und gesellschaftlichen Lebensbereichen. Diese Veränderungen standen jedoch, ganz abgesehen von ihren positiven und wünschenswerten Seiten, in einem engen Zusammenhang mit einer Welle zunehmender ernsthafter Probleme, wie Arbeitslosigkeit, Armut, Alkoholismus, Drogenabhängigkeit und Kriminalität.

Vor diesem Hintergrund ist der Staat in Polen dabei, viele dieser Aufgaben an kommunale Behörden und lokale Nichtregierungsorganisationen zu delegieren. Die Nichtregierungsorganisationen, meist Vereine oder Stiftungen, ergänzen insofern die institutionellen Strukturen des Staates als wichtige Teile der Zivilgesellschaft und des kollektiven Lebens.

Die »Barka-Stiftung für gegenseitige Hilfe« wurde 1989 von den beiden Psychologen Barabara und Tomasz Sadowki als Reaktion auf die zunehmend sozialen Probleme gegründet, die mit dem Transformationsprozess verbunden waren und noch immer sind. Sie wollten Bedingungen schaffen, in denen auch die Vergessenen und Ungewollten eine Chance auf Persönlichkeitsentwicklung und soziale Integration haben sollten. Im Rahmen ihres alternativen Hilfesystems sollten diese Menschen Möglichkeiten bekommen, ihre Leben neu zu ordnen, ihre beruflichen Fähigkeiten zu entwickeln und ihren Weg in die sozioökonomische Selbstständigkeit zu finden.

Die erste »Barka-Gemeinschaft « wurde 1989 in einem verlassenen Schulgebäude in Wladyslawowo im Westen der Stadt Poznan gegründet. Es bot ein Heim für 25 Menschen (»wrecks«) die zusammen mit der Familie Sadowski lebten und arbeiteten. Dieses erste Beispiel motivierte die weitere Entwicklung von gleichgerichteten sozialen Bewegungen, deren Ziel die fortlaufende gegenseitige Unterstützung und Hilfe war und motivierte Hilfebedürftige, aber auch informelle Gruppen und Nichtregierungsorganisationen, ähnliche Projekte zu starten.

Ziel der Barka-Stiftung ist es, sozial Ausgegrenzten Hilfe und Unterstützung zu geben, damit sie in die Lage kommen, ihr Leben eigenständig oder zusammen mit der Hilfe von anderen in die Hand zu nehmen, bei- spielsweise durch gegenseitige Hilfe, durch Erziehung oder durch selbstständiges Unternehmertum.

Heute hilft dieses Unterstützungssystem jährlich zirka 5.000 Personen, insgesamt existieren $30 »$ Barka-Gemeinschaften «, in denen 750 bedürftige Menschen leben und arbeiten. Meist handelt es sich um Selbstversorger-Bauernhöfe, die von Multigenerationsfamilien in die Stiftung eingebracht werden und auf der Idee der gemeinsamen Hilfe von Nachbarn und örtlichen Behörden fußen. Die Bewohnerinnen und Bewohner dieser Bauernhöfe müssen verschiedene Regeln respektieren und zum gemeinsamen Lebensunterhalt durch Feldarbeit, Mitarbeit im Haushalt, Erbringung von Dienstleistungen für Dritte etc. beitragen.

Arbeitslosigkeit ist inzwischen ein weitverbreitetes Problem. Die Arbeitslosenrate ist in Polen etwa zweieinhalb Mal so hoch wie im europäischen Durchschnitt. Vor diesem Hintergrund hat die Barka-Stiftung ein Projekt entwickelt, das der Entwicklung neuer Arbeitsplätze für Langzeitarbeitslose insbesondere durch Schaffung von Arbeitsplätzen auf dem Second-hand-Markt (Läden, Werkstätten, Bauernhöfe).

Ein wichtiger Bestandteil dieser Arbeit für von Exklusion bedrohte Gruppen sind die »Schulen zur sozialen Animation« (»SAS - Szkoly Animacji Socjalnej«). Sie basieren auf der Idee der Volksuntersität (»uniwersytety ludowe $\ll$ ). Diese nutzen die Erfahrungen und Kenntnisse ehemaliger Betroffener und versuchen in ihren Kursen Menschen in Schwierigkeiten zu zeigen, was man allein und gemeinsam - tun kann, um soziale Exklusionsprozesse zu verhindern oder zu bekämpfen. Auf diese Weise lassen sich zukünftige Lehrende und Animatoren für die Volksuniversitäten aus sich selbst heraus rekrutieren.

Die sozioedukativen Programme der Barka-Stiftung verbinden Sozialarbeit und Erziehung. Sie sind gegründet worden, um auf den zunehmenden Bedarf vom Arbeitsmarkt ausgeschlossener Gruppen nach Erziehung und Berufsbildung zu reagieren. Jedes Jahr nehmen einige hundert Menschen an diesen vielfältigen Programmen in Barka-Schulen teil.

Monika Marczak und Katarzyna Pawelek, Adam Mickiewicz University, Faculty of Educational Studies, Department of Resocialization, ul. Szamarzewskiego 89, 60-568 Pozna; College of Pedagogy and Administration, ul. Wisniowa 13, 61-477 Pozna E-Mail monikamarczak@tlen.pl E-Mail katarzyna.pawelek@inetia.pl 


\section{Der deutschen Sozialwirtschaft winkt die europäische Dividende}

Beispiele für schnelle und notwendige Erkenntnisgewinne durch Wissenstransfer, Kooperation und Konkurrenz können derzeit sein:

- Risk Management: Angesichts der jüngsten extremen Fälle elterlicher Gewalt gegen Kinder oder angesichts der in Zukunft massenhaft zu prüfenden Frage, ob ein alter Mensch noch zu Hause betreut werden kann, muss die Sozialarbeit Kriterien entwickeln, die es den Fachkräften erlauben, das mit der jeweiligen Entscheidung verbundene Risiko »valide« einzuschätzen. Solche empirisch gestützten Kriterien liegen in anderen Ländern (insbesondere England, vgl. Littlechild 2005) bereits vor und sollten dringend kritisch diskutiert und wo sinnvoll rezipiert werden.

- Ethische Codes: Eine breite Diskussion über angemessenes professionelles, methodisch richtiges und politisch korrektes Verhalten von Sozialarbeiterinnen und Sozialarbeitern findet in Deutschland noch nicht statt. Entwicklungen in Italien, England und Tschechien haben hier zur Formulierung expliziter (nationaler) Standards und zur Einführung von Berufsregistern geführt, die auch für die deutsche Sozialarbeit dringend erforderlich erscheinen.

- Evidence-based practice: Wirkungen und Qualität ausgewählter sozialer Dienste sind in verschiedenen Ländern teilweise außergewöhnlich intensiv beforscht worden (z. B. Italien: Betreuung psychisch Kranker, England: children and youth act). Das Wissen der Ergebnisse dieser Praxen erscheint für die Begründung eigener Praxis unverzichtbar.

- Sozialarbeit und Zivilgesellschaft: Die in Deutschland angestoßene Debatte nahm und nimmt zu wenig die Erkenntnisse wahr, die Ländern mit einer dementsprechenden längeren Tradition wie Spanien, insbesondere aber auch Frankreich (Chauvière 2004) bereits gemacht haben.

- Sozialarbeit und Ehrenamt: Die Einbeziehung von Ehrenamtlichen nimmt in vielen europäischen Ländern eine viel wichtigere strategische Position ein als in Deutschland. Professionelle Konzepte des »volunteer management « müssen rezipiert und in Bereichen (wie z. B. Flüchtlingshilfe, Altenarbeit) eingesetzt werden, wo die staatliche Unterstützung zunehmend zurückgeführt wird und es dadurch zu einer enormen Beeinträchtigung der Qualität kommt.

- Fundraising: Ähnliches gilt für Konzepte zur Akquise von Geld und anderen Ressourcen.

- Nutzerbeteiligung: Die Einbeziehung von Nutzern und Klienten in die Entscheidungsprozesse der jeweiligen Organisation steckt in Deutschland in den Kinderschuhen. Andere europäische Länder verfügen hier über vielfältige Erfahrungen, bis hin zur Einbeziehung von Klienten in die Ausbildung (Molyneux/Irvine 2004).

Peter Erath

risiken beigetragen, die sich kaum mehr rational steuern lassen (Erath/Sing 2005).

Wenn also eine moderne Gesellschaft ihre Teilsysteme, Organisationen und Professionen nur noch nach deren jeweiliger Funktion bewerten kann, die diese für die Gesamtgesellschaft erbringen, und andere Aspekte (wie Trägermotive, persönliche Einstellungen, mögliches Versagen anderer Teilsysteme etc.) aus formalen, rechtlichen, ethischen Gründen bei administrativen Entscheidungen nicht berücksichtigt werden können und dürfen, dann müssen sich die Organisationen darauf einstellen, dass der Wettbewerb zwischen den Einrichtungen letztendlich fast ausschließlich auf dem Feld der fachlichen Kompetenz ausgetragen wird. Dabei mitzuhalten erfordert jedoch eine deutliche Ausweitung der Theoriebildung, der Wissensgenerierung, der Forschung.

\section{Vorschläge}

Die angesichts dieser Situation veränderten Bedingungen können von den Einrichtungen nur dann gemeistert werden, wenn es ihnen gelingt, die dafür erforderlichen organisationalen, theoretischen und methodischen Voraussetzungen zu schaffen. Dies wird jedoch den Beteiligten nur gelingen, wenn sie ihr intellektuelles Argumentationsniveau deutlich zu steigern und nach außen die eigenen konzeptionellen Entwürfe und die praktische Performance entsprechend klug zu kommunizieren vermögen.

Dabei können die verschiedenen Anbieter - und das ist das eigentliche Problem - die Steigerung ihrer Leistungsfähigkeit kaum aus eigener Kraft bewältigen. Die materiellen und personellen Ressourcen im sozialen Bereich sind in der Regel zu knapp bemessen, nicht vorhanden oder werden nicht eingesetzt, um beispielsweise neue konzeptionelle Entwürfe zu erproben, zu implementieren und zu evaluieren, um Personal wirklich »exzellent « zu schulen und zu entwickeln oder um die eigene Arbeit wissenschaftlich abzusichern. Daher kommt es jetzt darauf an, sich mit anderen Organisationen (natürlich nicht mit den unmittelbaren Konkurrenten) zu vernetzen, um auf diese Weise Wissen kostengünstig generieren, transferieren und implementieren zu können. Aufgrund der großen Mängel der deutschen Sozialarbeit in der empirischen Forschung bietet sich hier die Zusammenarbeit zunächst vor allem auf europäischer Ebene an, der internationale Austausch von Erkenntnissen wird sich dann auf lange Sicht (ähnlich wie im Bereich der Ökonomik) von selbst einstellen.

Solche Vergleiche unter Gleichgesinnten erlauben die Möglichkeit zur Anschlussfähigkeit an national noch nie Gesehenes und fördern die Diskussion im eigenen Haus, ohne die Gefahr der öffentlichen Blamage. Zugleich bieten solche intrasystemischen Differenzen angesichts der 


\section{BEISPIELE GUTER PRAXIS: TSCHECHIEN \\ „Das Nest« kümmert sich sozialraumbezogen um Familien mit gefährdeten Kindern}

Das Projekt der Terrainassistenz für Familien mit gefährdeten Kindern - »Das Nest - iriklano Kher « - wurde von der Bürgervereinigung »Vzájemné souití« im Jahr 2005 gestartet. Auslöser war ein tragisches Ereignis in diesem Jahr: Ein neugeborenes Roma-Mädchen wurde im Mährisch-schlesischen Kreis gleich nach den Gratulationen durch die kommunalen Politiker der stillenden Mutter noch in der Entbindungsanstalt weggenommen und in einem Heim für Kinder bis drei Jahre in Ostrava-Zábeh untergebracht. Der Grund für die Anstaltsunterbringung des Mädchens: Die Familie hatte nicht rechtzeitig eine Babyausstattung für das Kind besorgt. Nach intensiven Bemühungen gelang es der Familie nach vier Monaten, das Sorgerecht für das Kind zurückzubekommen.

Ein weiterer Grund für den Start des Projektes war die Veröffentlichung eines Berichtes von Prof. Kevin Brown von der University of Birmingham, aus dem die schockierende Information zu entnehmen war, dass die Tschechische Republik die größte Anzahl von in Anstalten untergebrachten Kindern bis drei Jahre unter den europäischen Ländern hat.

Ziel des Projektes »Das Nest - iriklano Kher « ist es deshalb, die Zahl der in Erziehungsanstalten untergebrachten Kinder zu verringern und vorbeugend gegen die Kindesentziehung aus den Familien vorzugehen. Das Projekt wendet sich sowohl an Familien, die in eine Krisensituation geraten sind und die deshalb in der Gefahr stehen, dass ihre Kinder in Erziehungsanstalten kommen, als auch an Familien, deren Kinder sich bereits in Anstalten befinden und die sich um eine Rückkehr in die Familie bemühen.

Die Hilfe für die Familien wird in Form einer Terrainassistenz realisiert und konzentriert sich auf eine Lösung der Probleme in der Familie, auf die Hilfe bei Verhandlungen mit den Behörden, auf rechtliche Beratung (Sicherung von Beweismitteln), Vertretung beim Gericht, Assistenz, Einübung der Fertigkeiten, sozialrechtliche Beratung, humanitäre Hilfe sowie auf Vermittlung von Kontakten zwischen den Eltern und den Kindern in den Anstalten.

Derzeit beteiligen sich an diesem Projekt fünf Mitarbeiterinnen und Mitarbeiter des Terrainteams (zwei Sozialarbeiterinnen mit Hochschulabschluss, drei Terrainassistenten) und ein multidisziplinäres Team, das sich zusammensetzt aus Psychologen, Rechtsanwälten, Kinderärzten, Vertreter der Polizei der Tschechischen Republik (spezialisiert auf Kinder und Jugendliche). Seit dem Jahr 2005 konnten die genannten Teams 21 Familien helfen.

Die Tätigkeit der Teams richtet sich jedoch nicht nur auf die Unterstützung der gefährdeten Familien, ihr Ziel ist auch die Veränderung des Systems und eine geänderte Einstellung der Fachleute zur Unterbringung von Kindern in Erziehungsanstalten.

Einer der Ursachen, aufgrund dessen die Gerichte über die Unterbringung von Kindern in einer Erziehungsanstalt entscheiden, ist die Äußerung des Sozialarbeiters vom zuständigen Organ des sozialrechtlichen Schutzes für Kinder und Jugendliche (OSPOD), in der die Fachkraft eigentlich die Interessen des Kindes berücksichtigen soll. Problematisch ist jedoch, dass das Gesetz über den sozialrechtlichen Schutz für Kinder die Interessen des Kindes zu allgemein definiert. Daher trägt die Entscheidung, was das Beste für ein Kind ist, stets einen subjektiven Charakter. Eine Rolle in der Entscheidung spielen auch die Stellungnahmen der Personen, die darüber entscheiden, ob das Kind in der eigenen Familie aufwachsen wird oder nicht. Die Stellungnahmen selbst können sich unter dem Einfluss der Umgebung verändern, deshalb sind die Aktivitäten des Projektes auf eine positive Beeinflussung gerichtet. Zur Veränderung des Systems und der Stellungnahmen zur Unterbringung der Kinder in Erziehungsanstalten wurden einige runde Tische veranstaltet und es sind weitere Veranstaltungen zur Einwirkung auf die Fach- und Laienöffentlichkeit geplant:

Im September 2005 wurde der erste runde Tisch in Ostrava mit dem Thema veranstaltet: »Wie kann die Zahl der Kinder in Anstalten gesenkt werden?«, zu dem eingeladen waren die Vertreter der örtlichen nichtstaatlichen gemeinnützigen Gesellschaften, des Mährisch-schlesischen Kreises, Sozialarbeiterinnen und Sozialarbeiter von der Abteilung des sozialrechtlichen Schutzes der Kinder und Jugendliche, Vertreter des Arbeits- und Sozialministeriums, der Universitäten (Universität Ostrava, Masaryk-Universität Brno), der Polizei u. a. Hier wurden die Gründe für die Unterbringung in der Anstaltspflege diskutiert. Die Teilnehmer einigten sich auf vier Gründe, und zwar:

- mangelhafte Koordination, weil die Problematik der Anstaltserziehung der Kinder nicht von einem Ressort gesteuert wird

- zu wenig Personal, was zum Burnout-Syndrom führt und zu der Einstellung, dass die Anstaltserziehung das Beste für das Kind ist

- unzureichende Zusammenarbeit zwischen den interessierten Beteiligten

- Probleme im Wohnungsbereich schwächen die Funktion der Familie

Bei der zweiten Tagung des runden Tisches, die Ende Februar 2006 in Ostrava veranstaltet wurde, widmeten sich die Teilnehmer der Ausarbeitung einer Methodik der Fallkonferenzen, die zu einem Beispiel werden könnten, wie man an der Lösung der Probleme auf der Basis des Prinzips von der gleichberechtigten Partnerschaft zusammenarbeiten kann. Das ausgearbeitete Modell wurde bei der Tagung den Vertretern des Arbeits- und Sozialministeriums ausgehändigt, das sie weiter ausarbeiten und den einzelnen Abteilungen des sozialrechtlichen Schutzes für Kinder und Jugendliche (OSPOD) und den beteiligten Partnern zur Verfügung stellen werden.

Alle genanten Bemühungen des Projektes zielen auf die Erfüllung der Bestimmungen des Artikels Nr. 7 vom Abkommen über die Rechte des Kindes ab. Die Rechte besagen, dass jedes Kind das Recht hat, seine Eltern zu kennen und das Recht auf eine Betreuung durch diese. Die Tätigkeit der Vereinigung wird insbesondere durch das Daphne-Programm der Europäischen Kommission sowie die Stiftung der Bürgergesellschaftsentwicklung, das tschechische Arbeits- und Sozialministerium und den Magistrat der Stadt Ostrava unterstützt.

Anna Krausová und Hana urovcová, Universität Ostrava, Bürgervereinigung Vzájemné souití (Gemeinsames Zusammenleben), Das Nest - iriklano Kher - Terrainassistenz der gefährdeten Familien
E-Mailvzajemne.souziti@tiscali.cz
E-Mailanna.krausova@osu.cz 
Tatsache, dass »die Anderen « oftmals auch nicht viel besser sind als wir, die Möglichkeit, Abschied zu nehmen von den teilweise immer noch rigiden Codes der Sozialarbeit wie richtig und falsch, gut und böse, konservativ und kritisch. Sie erlauben den Übergang zu stärker konstruktivistischen und relativistischen Sichtweisen, wie geeigneter und weniger geeignet, passender und weniger passend, erfolgversprechender und weniger erfolgversprechend, kritischer und weniger kritisch.
Auf der Ebene der Organisationen können jetzt drei Lösungswege diskutiert und etabliert werden:

- Unter "good practice« versteht man erfolgreiche Lösungen unter Beachtung anerkannter Standards, wobei es hier häufig mehrere Lösungen geben kann. Entscheidend kommt es für eine Organisation oder einen Mitarbeiter darauf an, für sich selbst die »gute« Lösung zu finden und dann entsprechend zu praktizieren.

\section{BEISPIELE GUTER PRAXIS: NIEDERLANDE}

\section{Empowerment-Projekte für marokkanische Mädchen und junge Frauen beziehen den Islam mit ein}

Die marokkanische Gemeinschaft ist einer der größten Minderheitengruppe in den Niederlanden. In jüngster Zeit ist daher der Entwicklung junger Marokkanerinnen und Marokkaner in Schule, Hochschule und im Beruf eine größere öffentliche Aufmerksamkeit gewidmet worden. Dabei handelte es sich häufig um Projekte, die vom Prinzip des Empowerments ausgehen.

Bislang wurde dabei jedoch die Rolle, die der Islam spielt, zu wenig beachtet. Daher wurden jetzt MädchenProjekte gestartet, die von der These ausgehen, dass dem Islam im Rahmen solcher Empowerment-Prozessen eine wichtigere Rolle eingeräumt werden muss. Angenommen wird, dass nur er in der Lage ist, die jungen Heranwachsenden angemessen mit emotionalen Stärken und persönlichem Stolz auszustatten.

Das ROC-Projekt, ein von dieser Annahme ausgehendes Projekt in Amsterdam, zielt auf marokkanische Mädchen und junge Frauen von 18 bis 22 Jahren und nutzt vor allem folgende Methoden:

- Interkultureller Dialog: Dieser dient dazu, die kulturelle Vielfalt der einzelnen Person wie auch die Dynamik und Flexibilität von Kulturen kennen zu lernen. Er hilft den Einzelnen die »Vision des eigenen Lebens « vor dem Hintergrund sozialer Vielfalt und Respekt vor anderen zu entwickeln.

- VUT-Modell: Dieses Modell basiert auf drei Prinzipien: Schau nach vorne, tu es und schaue zurück! Es will jungen Menschen die Bedeutung von Selbstevaluation und Feedback vermitteln und aktiviert dabei das Denken über sich selbst.

- »Quality game«: Diese Methode soll den Mädchen und jungen Frauen zeigen, welche wichtige qualitative Rolle die Erfahrungen der Großeltern, Eltern etc. im Familienleben auf welche Weise spielen.

- Systemische Theorie: Im Rahmen beispielsweise eines Genogramms soll den Mädchen und jungen Frauen bewusst werden, woher sie kommen. Außerdem sollen sie positiv begreifen, welches familiäre Netzwerk sie umgibt und wie viel soziale Unterstützung damit verbunden ist.

Insgesamt zielt das Projekt auf die Beantwortung dreier Fragen: Wer bin ich? Was möchte ich erreichen? Von woher bekomme ich die Kraft, um vorwärtszukommen? Logischerweise spielt im Rahmen einer solchen Vorgehensweise der Glaube eine wesentliche Rol- le. Mädchen können beispielsweise Stärken aus der Aufforderung Mohammeds gewinnen, Frauen sollten gut behandelt werden. Denn diese Mädchen und Frauen haben meist erstaunliche emotionale Qualitäten, aber nur ein geringes Selbstwertgefühl.

Im Rahmen des Empowerment-Prozesses setzen sie sich mit ihrer Vergangenheit und mit ihrer Realität auseinander. Auf diese Weise lernen sie neue Aspekte ihrer Persönlichkeit kennen, insbesondere den, aktiv zu sein und eigene Entscheidungen zu treffen. Zugleich lernen sie, mit ihren Familien besser zu kommunizieren, was zu Veränderungen in den Mustern der familiären Kommunikation und des Verhaltens führen kann.

Ein anderer Aspekt im kulturellen Kontext des Islams sind die Familienehre und die Gruppenabhängigkeit. Auch hier können sich im Rahmen des EmpowermentProzesses Veränderungen ergeben, weil die Mädchen und Frauen jetzt deren Hintergrund kennen und damit besser mit den jeweiligen Themen umgehen können.

Eine Evaluationsstudie des ROC-Projekts ergab, dass die meisten Mädchen die Ideen des Islams in einer bewussten Weise aufgreifen und so in die Lage versetzt werden, eine positive Lebensweise zu entwickeln, in der es ihnen schließlich gelingt, ihr persönliches Potenzial voll zu nutzen. Deutlich wurde in der Studie auch, dass die meisten Eltern in der Regel keine Einwendungen gegen das ROC-Projekt haben, da es auf dem Islam basiert. Die Schlussfolgerung der Autoren der Studie ist daher:

»Wenn die Ergebnisse so positiv sind, dann sollten die auf dem Islam basierenden Empowerment-Projekte in den Niederlanden, einem Land in dem die Mehrheit aller Minderheiten muslimischen Glaubens ist, weiter ausgedehnt werden. Diese Kurse sind so sehr effektiv, weil sie sich an den kulturellen Hintergrund und and den religiösen Praktiken der Mädchen orientieren. Mädchen finden darin eine spirituelle Ressource um ihr Leben in die Hand zu nehmen. Die Verbesserungen und Fortschritte, die sie in den Kursen machen, entstehen und bleiben in ihrem Inneren. Insofern können sie immer wieder auf diese Stärken zurückgreifen, die Quelle des Empowerments ist in ihnen!«

Dr. Leonie van der Valk, Dr. Anja van Heelsum, Universiteit van Amsterdam, International School for Humanities and Social Sciences (ISHSS), The Role of Islam in the Empowerment Process of Adolescent Moroccan Girls in the Netherlands.

E-Mail ishss@uva.nl 
- Beim »Best-practice-Modell« wird davon ausgegangen, dass Verfahren und Methoden, die sich in einer Firma bestens bewährt haben, in ihrer Gesamtheit, also einschließlich von Strukturen etc., übernommen werden. Der eigentliche Gewinn besteht darin, die beste Lösung nicht selbst erst entwickeln (und diese Entwicklung finanzieren) zu müssen, sondern tatsächlich sich als effizient etc. erweisende Konzepte in Gänze übernehmen zu können.

- Ausgelöst durch Entwicklungen in der Medizin wird heute auch zunehmend das Modell der »evidence-based practice« diskutiert: Dieses geht davon aus, dass über eine systematische Auswertung von wissenschaftlichen Studien und Forschungsergebnissen konkrete Entscheidungshilfen für die Anwendung richtiger und effektiver Behandlungsmethoden gegeben werden können. Idealerweise würde sich so ein berufsspezifisches Wissen entwickeln, innerhalb dessen es möglich wäre, Fallproblematik und Lösungsweg direkt aufeinander zu beziehen (Bilson 2005, Wendt 2005).

\section{Folgerungen für das Management}

Soziale Einrichtungen in Deutschland können die mit dieser neuen Sichtweise verbundenen Herausforderungen nur im Rahmen eines fundamentalen Wandels ihres Selbstverständnisses und der daraus erwachsenden Folgerungen für Organisation und Management bewältigt werden, wie zum Beispiel:

- Trägerschaft: Die freien Träger sozialer Dienste sind Anbieter unter anderen und werden auf Dauer keine (politische) Sonderbehandlung mehr genießen. Sie lassen sich daher langfristig nur über eine hohe fachliche und organisationale Kompetenz legitimieren, hoch bedeutet dabei immer: europäisch und international anschlussfähig.

- Trägerleitbild: Die strategische und praktische Umsetzung des Trägerleitbildes kann nur auf der Trägerebene selbst erfolgen. Die Hoffnung, Leitbilder ließen sich im beruflichen Alltag operationalisieren, beruht nicht nur

\section{BEISPIELE GUTER PRAXIS: ITALIEN}

\section{"Libera« macht beschlagnahmtes kriminelles Ver- mögen für soziale Zwecke nutzbar}

Soziale Kooperativen haben in Italien eine lange Tradition und sind inzwischen in vielen sozialen Feldern gut etabliert. Sie repräsentieren einer der innovativsten Erfahrungen innerhalb des Non-Profit-Sektors.

Dabei lassen sich zwei Haupttypen von sozialen Kooperationen unterscheiden: Auf der einen Seite gibt es Kooperativen, die von Professionellen gegründet wurden, um soziale Dienste von öffentlichem Interesse zu organisieren. Auf der anderen Seite wurden sie gegründet, um Arbeitslosen eine Möglichkeit zu geben, einer konkreten Beschäftigung nachzugehen. Solche Kooperationen können sehr kurzfristig angelegt sein und seitens der Betroffenen als vorübergehende Möglichkeit wahrgenommen werden, um sich weiter zu bilden und damit teilweise auch zu resozialisieren. Oder sie können dazu dienen, die Betroffenen, die stärker beispielsweise gesundheitlich eingeschränkt sind, vor Marginalisierung und vollständiger sozialer Exklusion zu bewahren.

In letzter Zeit haben sich beide Formen sozialer Kooperativen zunehmend differenziert und sind zu qualifizierten Partnern der regionalen Gesundheits- und Sozialbehörden bei der Sicherung der Versorgung der Bevölkerung mit sozialen Diensten geworden. Sie sind nun auch strukturell und rechtlich als politische Einrichtungen anerkannt worden, denen man eine große Autonomie in Bezug die konzeptionelle und methodische Gestaltung ihrer Arbeit einräumt.

Gewichtige Faktoren dieser Organisationen sind ihre Flexibilität und Innovationsbereitschaft, oftmals reagieren sie auch auf spezifische Bedarfe, denen die lokalen oder nationalen Behörden nicht länger angemessen und gerecht werden oder für die kein Geld zur Verfügung steht. Zudem spielen sie, zusammen mit den an- deren Körperschaften im Non-Profit-Sektor eine wichtige kulturelle und politische Rolle.

Soziale Kooperativen haben mitgeholfen Vereinigungen einzurichten, die wichtige soziale Aufgaben effizienter erfüllen. Ein interessantes und bedeutsames Beispiel dafür ist die Vereinigung »Libera«, gegründet im Jahre 1995, die sich zum Ziel setzt, alle Bemühungen der Zivilgesellschaft gegen kriminelle Vereinigungen zu koordinieren.

Auf der Basis eines 1996 geschaffenen Gesetzes ist es möglich geworden, den Besitz krimineller Vereinigungen nach der Beschlagnahmung sozialen Zwecken zuzuführen. Vor diesem Hintergrund entstand eine große Zahl an sozialen Kooperativen, die sich zum Ziel gesetzt hat, die konfiszierten Ländereien und Gebäuden zu verwalten, vor allem im landwirtschaftlichen Sektor - und nicht nur in Süditalien.

Damit ist nicht nur eine Möglichkeit entstanden, neue Jobs zu schaffen und damit die wirtschaftliche Situation vieler Menschen zu verbessern, zugleich sind zivilgesellschaftliche Bemühungen entstanden, die darauf abzielen, den repressiven Kampf des Staates gegen das organisierte Verbrechen durch einen kulturellen Kampf der Prävention zu unterstützen. So ist »Libera « (sie umfasst insgesamt zirka 10.000 lokale und nationale Gruppen) nun sehr stark in erzieherische und kulturelle Aktivitäten involviert, die zum Ziel haben, die Kultur und den Einfluss krimineller Organisationen in ganz Italien zurückzudrängen.

Soziale Arbeit wird hier verstanden als professionelle Unterstützung und Empowerment von Menschen, die von sozialer Exklusion und Marginalisierung bedroht sind., und als politische Form der Einflussnahme auf die sozialen Beziehungen von Menschen und Gruppen mit ihren Regeln und Werten.

Rino Fasol, Universität Trento

E-Mail rino.fasol@soc.unitn.it 
auf einem logischen Fehlschluss, sondern auch auf Annahmen, die die Autonomie fachlicher Entwicklungen und Diskurse, wie sie in anderen Ländern schon lange auf hohem Niveau geführt werden, völlig unterschätzen.

- Performance: Anbieter sozialer Dienstleistungen bieten entweder sehr gute Qualität oder sie werden von den Kommunen kontrolliert, auditiert, überwacht etc. Auch die Kommunen werden sich dabei an den Standards europäischer oder internationaler »best practice« orientieren.

- Wissenstransfer: Träger sozialer Einrichtungen werden in Zukunft nicht ohne systematisches Wissensmanagement auskommen und dazu eigene Abteilungen für Forschung, Entwicklung, Implementation, oder »Excellence center « sowohl schaffen als auch miteinander verknüpfen müssen. Die Herstellung von Anschlussfähigkeit an das Wissenschaftssystem, das von Natur aus international ist (auch wenn dies in Deutschland in der Sozialen Arbeit lange nicht der Fall war), wird dafür eine wichtige Voraussetzung sein.

- Qualitätsnachweise: Die Einrichtung trägereigener Gütesiegel, Akkreditierungsverfahren etc. ist ungeeignet, Vertrauen in die versprochene Leistungsfähigkeit herzustellen. Gerade nichtstaatliche Einrichtungen müssen sich an höchsten fachlichen Standards orientieren und sich insofern den entsprechenden, als hochrangig eingestuften (weil: europäisch und international abgesicherten) Qualitätssicherungsverfahren selbstbewusst stellen.

- Management: Managerinnen und Manager in der Sozialwirtschaft benötigen ein hohes Maß theoretisches und konzeptionelles Wissen aus der Sozialarbeitswissenschaft. Wenn Sozialarbeiterinnen und Sozialarbeiter »etwas tun, was man (eigentlich) nicht kann « (Luhmann), dann kommt dem theoretischen Design und der empirischen Absicherung von Hilfeleistungen eine fast genauso wichtige Funktion zu, wie der organisationalen Steuerung und der konkreten Erbringung.

- Personalentwicklung: Maßnahmen zur Personalauswahl und Personalentwicklung kommen in einer hochdifferenzierten Sozialarbeit höchste Priorität zu. Insbesondere jungen Mitarbeiterinnen und Mitarbeiter müssen Karrieren eröffnet und weit mehr Entwicklungschancen als bislang eingeräumt werden. Die Europäisierung und Internationalisierung des Denkens muss bereits im Studium angelegt werden.

- Gesellschaftspolitische Integration: Managerinnen und Manager sozialer Dienste dürfen die Einbeziehung Ehrenamtlicher nicht nur als Notwendigkeit, sondern müssen sie als strategische Aufgabe zur Optimierung der Integration ihrer Organisation in die Gesamtgesellschaft verstehen.

\section{Schlussbemerkung}

"Helfen ist eine Kommunikation, die darüber informiert, dass ein Defizit besteht, mitteilt, dass dieses Defizit behoben werden soll, und verständlich macht, dass zwischen dem Bestehen eines Defizits und seiner Behebung nicht etwa ein kausal verlässlicher, sondern ein höchst kontingenter Zusammenhang besteht. «

Dirk Baecker 1994, S. 99
Wenn das Zitat von Baecker zutrifft, dann muss die Soziale Arbeit danach streben, die »kontingenten«, ihr als System zur Verfügung stehenden konzeptionellen und methodischen Möglichkeiten entwickeln und steigern. Eine nationale Sozialarbeit ist dazu heute nicht mehr in der Lage. Wenn zudem auch ethisch betrachtet nur die beste Form der Hilfe und Betreuung - angesichts der Leiden der Betroffenen - adäquat sein kann, so erscheint die Europäisierung und Internationalisierung der Sozialarbeit nachgerade eine Pflicht aller Anbieter und Beschäftigten in diesem Bereich zu sein.

\section{Literatur}

Baecker, D. (1994): Soziale Hilfe als Funktionssystem der Gesellschaft. In: Zeitschrift für Soziologie 23, S. 93-110.

Bilson, A. (Ed.) 2005: Evidence-based practice in social work. London. Whiting \& Birch.

Chauvière, M. (2004): Le travail social dans L'action publique. Sociologie d' une qualification controversée. Paris. Dunod.

Erath, P. (2006): Sozialarbeitswissenschaft. Eine Einführung. Kohlhammer. Stuttgart.

Erath, P., Sing, H. (2005): De- and Reconstruction of Social Welfare and Social Work in Germany. In: Littlechild, B., Erath, P., Keller, J. (Eds.) De- and Reconstruction in European Social Work. ISIS. Eichstätt, S. 159-174.

Littlechild, B. (2005): Gefahren und Nutzen von Risikobewertung und Risikomanagement in der Sozialen Arbeit. Sozialmagazin 30, S. 31-39.

Molyneux, J., Irvine, J. (2004): Service User and Carer Involvement in Social Work Training: a Long and Winding Road? Social Work Education. Vol 23, No. 3, S. 293-308.

Wendt, W. R. (2005): Maßgaben für eine gute Praxis. Evidenzbasierung Sozialer Arbeit. In: Blätter der Wohlfahrtspflege 152. S. $168-173$.

\section{Vorsprung durch Kompetenz!}

\section{Masterlehrgang European NGO Management}

- Berufsbegleitend in 4 Semester zum Master (Msc.)

- Spezialisierungen: Rettungswesen, Pflege, Krisenmanagement, Bildung

- Top Referenten

- Internationale Hochschule - international anerkannter Abschluss

- Zulassungsvoraussetzung - Details unter www.imt-kufstein.at

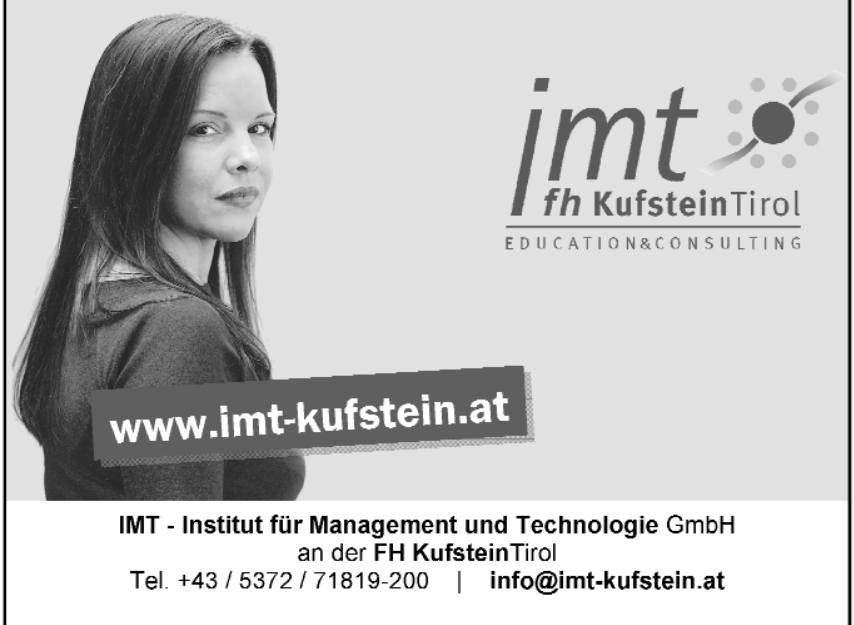

